\title{
Automatisiertes Hochdurchsatz-Verfahren zur reproduzierbaren Messung des Energieertragspotenzials von Biomassen
}

\author{
Ottmar Schläfer, Theodore Onyeche, Michael Sievers
}

\author{
Clausthaler Umwelttechnik-Institut GmbH (CUTEC), Clausthal-Zellerfeld/Deutschland, \\ Leibnizstraße $21+23$
}

\section{Zusammenfassung}

Die Entstehung des Methans als Energieträger ist bei der anaeroben Vergärung stark abhängig von der Zusammensetzung der Biomasse, den eingesetzten Vorbehandlungsmethoden sowie von der Art und Menge der Zuschlagstoffe. Die Substratkombination und Verfahrensweise von Biogasanlagen, die zur maximalen Biogasausbeute führen, lassen sich nur durch Laborversuche wirtschaftlich und zeitnah ermitteln. Da bisher am Markt keine für groß angelegte Versuchsreihen geeigneten Geräte verfügbar waren, war es das Ziel des CUTEC-Institutes, ein solches System zu entwickeln. Für die Gasvolumenmessung beinhaltet das neue Messverfahren drucküberwachte Gasmesszellen. Die Methan- und die Kohlendioxidkonzentration werden mit Hilfe von Infrarotsensoren online gemessen. Wasserdampf wird aus dem Messgas kondensiert und in den Gärreaktor zurückgeführt, die Gasvolumina werden auf Standardbedingungen normiert, das Energieertragspotenzial wird automatisch berechnet. Das neue Messverfahren zeichnet sich aus durch: (1) Hohen Probendurchsatz, (2) hohe Auflösung ( $\mu 1$-Bereich), (3) gute Reproduzierbarkeit und (4) hohe Wirtschaftlichkeit.

\section{Einleitung und Aufgabenstellung}

Seit Ende 2010 sind in Deutschland rund 6.000 Biogasanlagen mit einem Flächenbedarf von ca. 0,75 Mio. Hektar in Betrieb. Mit einer gesamten installierten elektrischen Leistung von rund 2.300 Megawatt (MW) werden bereits mehr als $10 \%$ der rund 40 Mio. Privathaushalte in Deutschland mit Strom aus Biogas versorgt. Trotz dieser Erfolge der vergangenen Jahre scheint es fraglich, ob in den folgenden 10 Jahren die durch die Bundesregierung selbst gesteckten Ziele für die Biogas-Erzeugung und -Einspeisung erreicht werden können. Danach sollen bis zum Jahre 2020 mindestens 7\% (6 Mrd. $\mathrm{m}^{3}$ ) Erdgas durch Biogas ersetzt werden. Zum aktuellen Zeitpunkt werden maximal 0,5 Mrd. $\mathrm{m}^{3}$ Biogas eingespeist, wobei ca. $75 \%$ der insgesamt in Deutschland für die Biogaserzeugung zur Verfügung stehenden Fläche (ca. 2 Mio. ha) bereits verbraucht sind. Neben der Anpassung politischer Vorgaben (EEG 2012) ist die Biogaswirtschaft in ihrer gesamten Wertschöpfungskette daher verstärkt auf Effizienzfortschritte angewiesen. Eine wesentliche Voraussetzung zur Verbesserung der Leistungsfähigkeit von Biogasanlagen ist die Verfügbarkeit von leistungsfähigen Messsystemen zur zuverlässigen Ermittlung des Biogas-/Energieertragspotenzials.

Nach VDI 4630 bieten diskontinuierliche Gärtests die derzeit sicherste Möglichkeit, das Biogaspotenzial eines Substrates mit einem vertretbaren Aufwand zu bestimmen. Da bisher am Markt keine automatisierten, für groß angelegte Versuchsreihen geeigneten Geräte verfügbar sind, war es das Ziel, ein Messsystem zur automatischen Erfassung der Biogasmengen für die anaerobe Vergärung von Biomasse zu entwickeln. Die Gasentwicklung bei der Vergärung von Biomasse in diskontinuierlichen Laborversuchen erfolgt entsprechend dem biologischen Abbau der organischen Verbindungen und der Zunahme der Gas produzierenden Zellmasse in einem „S“-förmigen Kurvenverlauf. Nach einem steilen Anstieg der Gasentwicklung bis zu einem Maximum, folgt ein Abflachen der Gaskurve durch 
den Rückgang der Gasentwicklung zum Ende der Vergärung. Daraus ergibt sich ein Volumenstrom, der im Laufe der Vergärung um mehrere Zehnerpotenzen variiert und zum Ende der Vergärung nur noch wenige $\mathrm{ml} / \mathrm{d}$ beträgt. Der biologische Prozess darf bei der Gasmessung nicht beeinflusst werden. Wesentlich ist daher die Konstanthaltung der für den anaeroben Vergärungsprozess erforderlichen Milieubedingungen. Die gestellten Anforderungen an das Messgerät und die einzelnen Baugruppen sind in Bild 1 wieder gegeben.

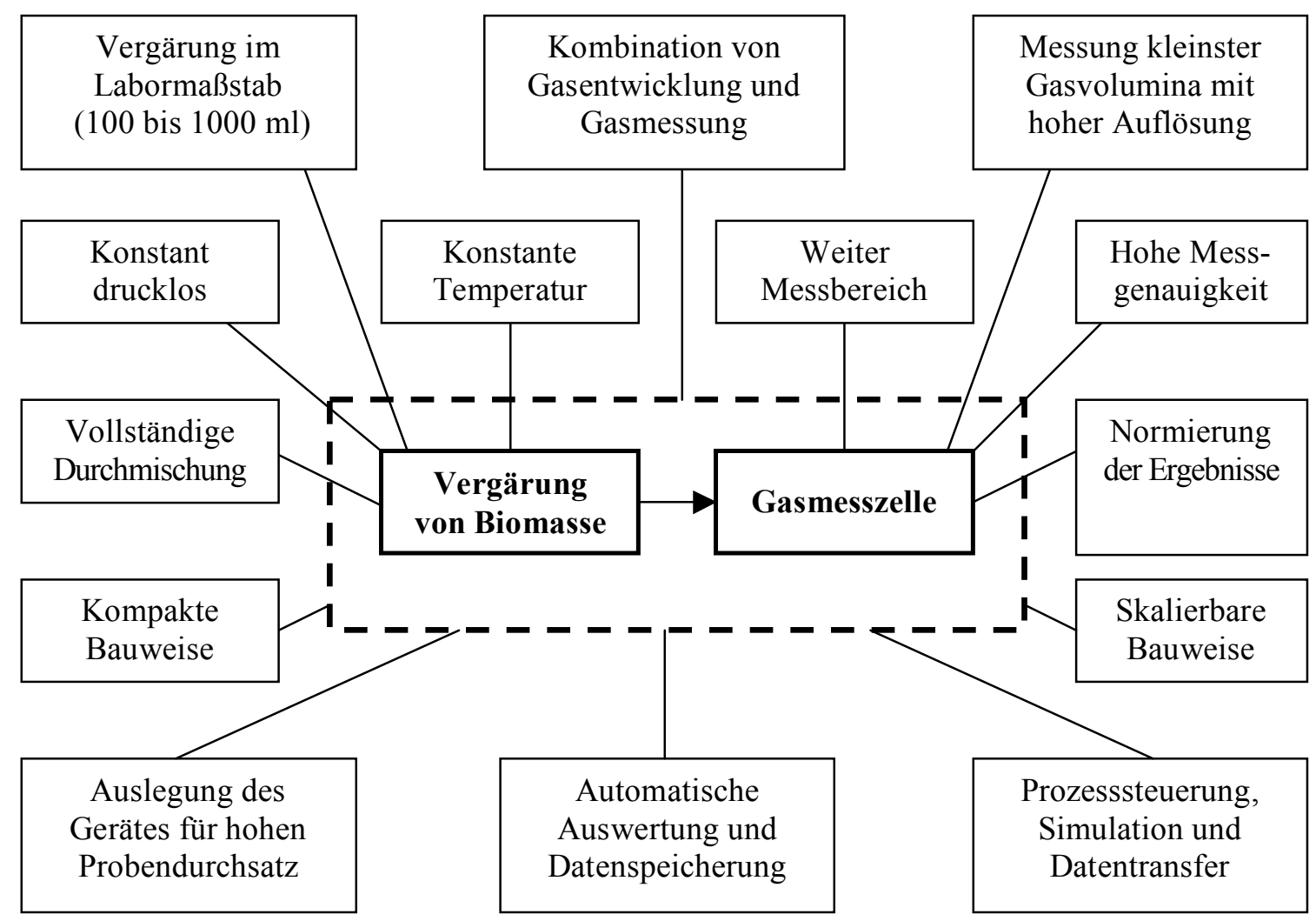

Bild 1 Anforderungen an das zu entwickelnde Gasmessgerät.

\section{Ergebnisse}

Kernstücke des Messverfahrens bilden gasdicht abgeschlossene prozessorgesteuerte Miniaturreaktoren zur reproduzierbaren Vergärung von Biomasse und mit dem Gasraum der Reaktoren in Verbindung stehenden Gasmesszellen. Diese Messzellen sind mit empfindlichen Innendruck- und Differenzdrucksensoren ausgestattet, welche bereits Druckänderungen, die durch die Entstehung von weniger als $1 \mu \mathrm{l}$ Biogas hervorgerufen werden, detektieren können. Beim Erreichen von voreinstellbaren Druckschwellen im 1hPa-Bereich werden die gebildeten Volumenelemente durch automatisierte Ventilsteuerungen aus dem System ausgeschleust. Gleichzeitig werden die Gaskonzentrationen von Methan und Kohlendioxid durch integrierte Zweistrahl-Infrarotsensoren innerhalb der Gasmesszellen bestimmt. Der Wasserdampf wird vorher aus dem Messgas kondensiert und in den Gärreaktor zurückgeführt. Das Messverfahren ist automatisieret, die Gasvolumina können automatisch auf Standardbedingungen normiert werden, das Energieertragspotenzial der Biomasse (berechnet aus Gasvolumen und Methanertrag) kann direkt angezeigt werden. Bild 2 verdeutlicht den prinzipiellen Aufbau der Messapparatur. 


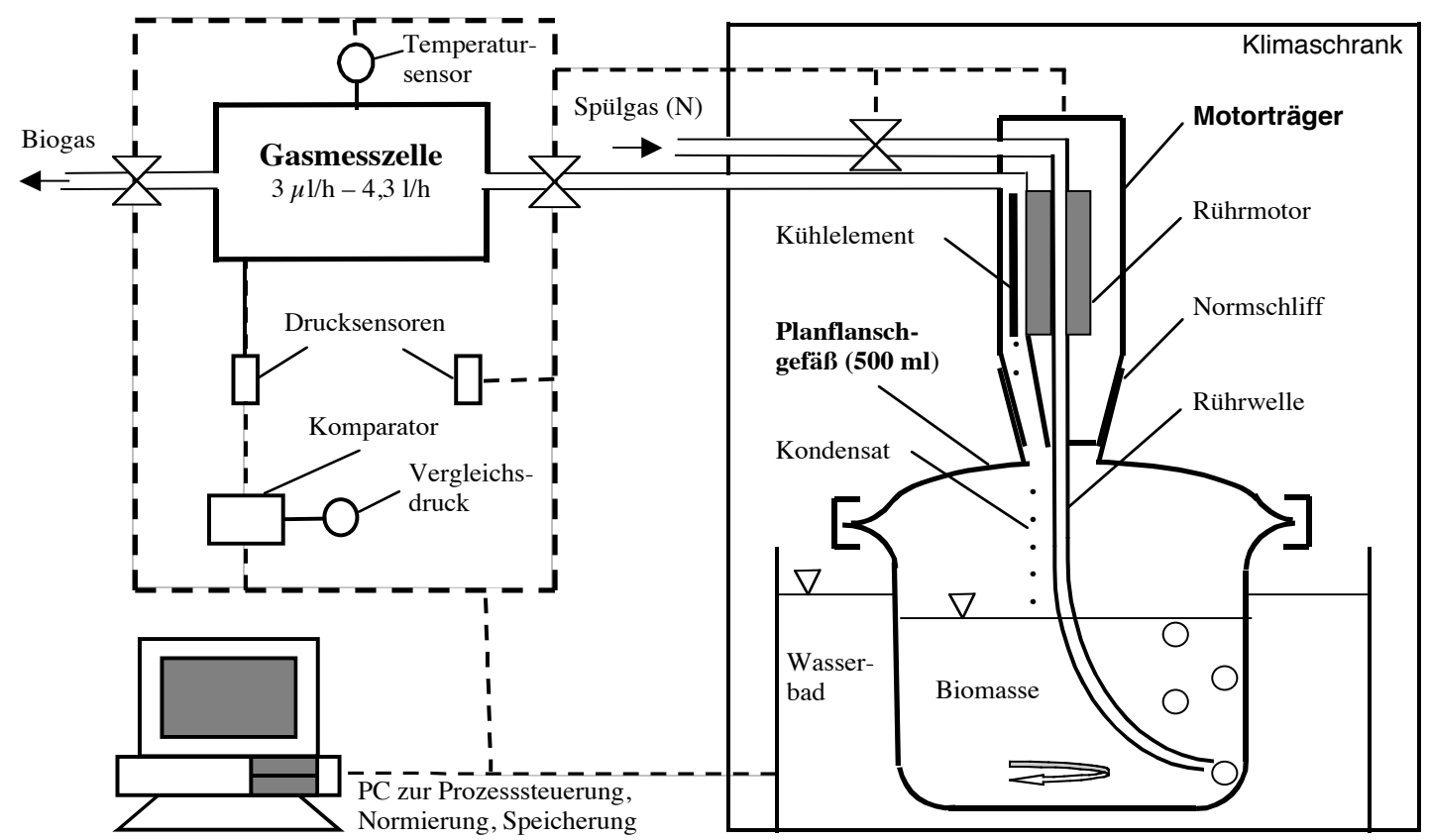

Bild 2 Aufbau und Funktion der Messapparatur.

\section{Volumenmessung (Reproduzierbarkeit)}

Eine hohe Reproduzierbarkeit (hohe relative Genauigkeit, geringe Fehlerschranken) bei der Biogaspotenzialmessung ist eines der wesentlichen Ziele bei der Entwicklung des standardisierten Messverfahrens. Damit sollen auch kleinste Unterschiede im Biogaspotenzial der Substrate noch sicher differenziert werden können. Die Standardisierung des Vergärungsprozesses muss eine möglichst geringe Streuung der Parallelansätze untereinander zulassen, aber auch eine gute Reproduzierbarkeit von Ergebnissen bei einer Wiederholung der Versuche zu einem späteren Zeitpunkt. Die Ergebnisse zeigten, dass bei drei im Abstand von 7 Tagen hintereinander angesetzten Vergärungsversuchen bei Dreifachbestimmungen lediglich Abweichungen von 2\% auftraten. Im Bild 3 sind die Versuchsergebnisse dargestellt. Damit waren die Messfehler der Apparatur bereits kleiner, als die durch den Impfschlamm verursachten Einflüsse. Dies hat im CUTEC dazu geführt, dass der Impfschlamm ebenfalls standardisiert wurde.

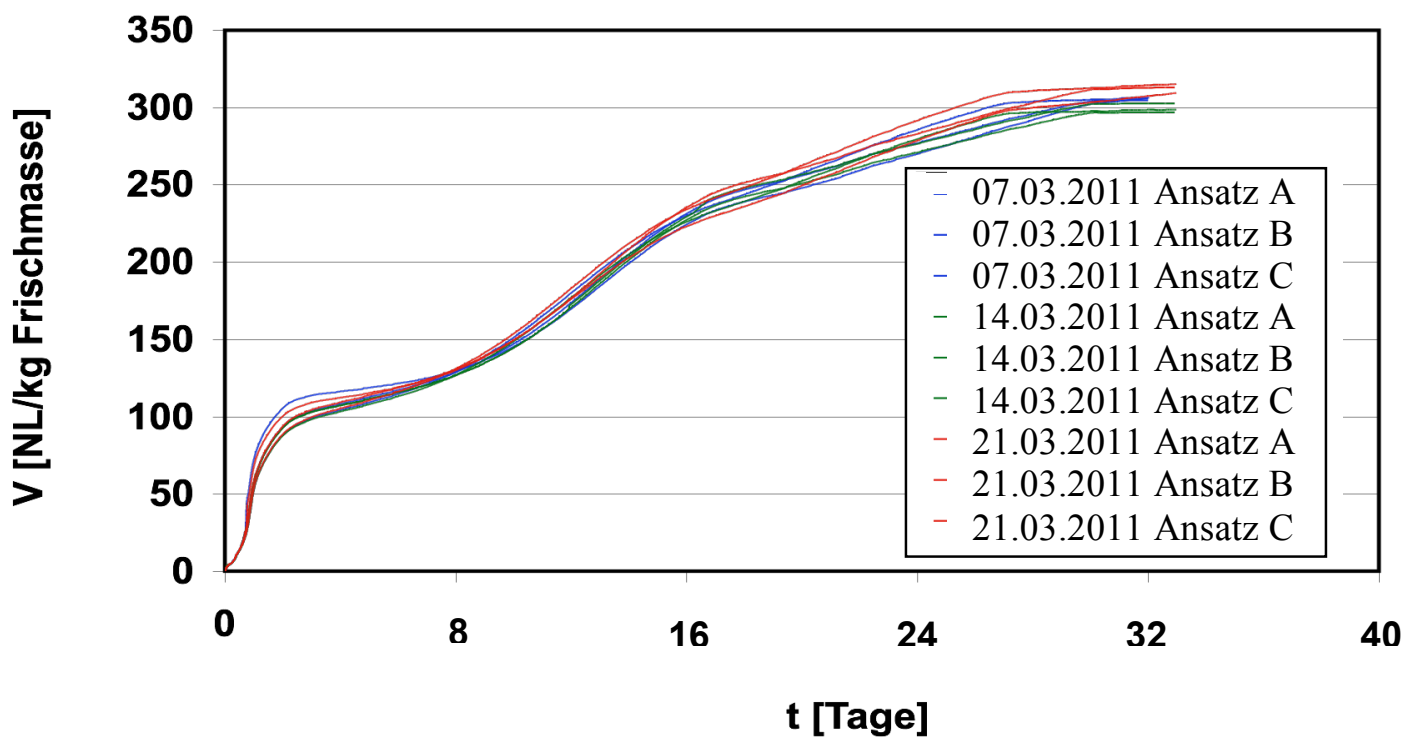

Bild 3 Reproduzierbarkeit des Gasertragspotenzials (Gesamstandardabweichung 5,35 NL/kg oTR). 


\section{Konzentrationsmessung}

Die Gaskonzentrationen von Methan $\left(\mathrm{CH}_{4}\right)$ und Kohlendioxid $\left(\mathrm{CO}_{2}\right)$ wurden mit Hilfe der InfrarotGassensoren kontinuierlich aufgezeichnet. Im Bild 4 sind beispielhaft normierte Gaskonzentrationen und die Addition beider Kurven wieder gegeben.

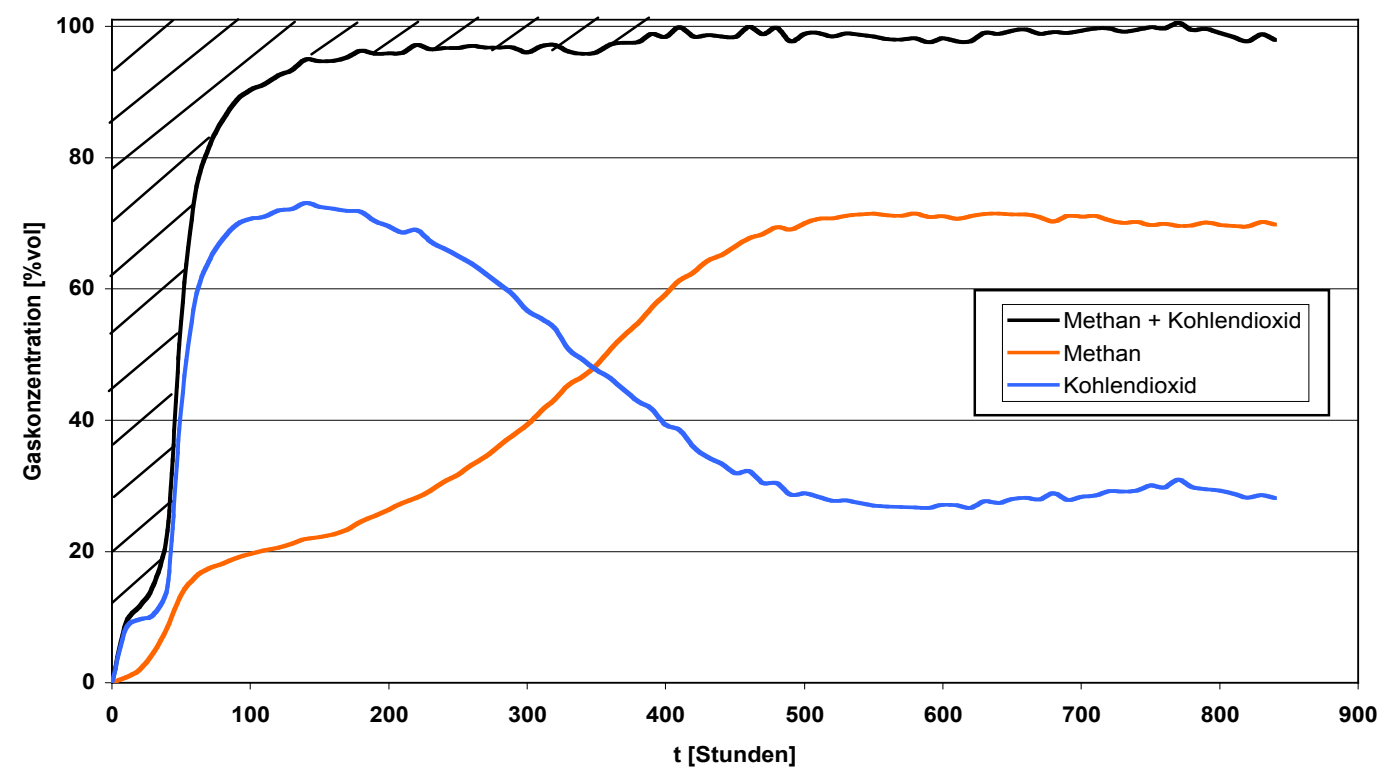

Bild 4 Kontinuierlich gemessene Methan- und Kohlendioxidkonzentrationen.

$\mathrm{Zu}$ Beginn der Vergärung wird das System mit Stickstoff gespült, um anaerobe Bedingungen in den Vergärungskolben sicher zu stellen. Dieser Stickstoffanteil wird erst allmählich aus dem Messgas verdrängt (siehe Schraffur). Der Methananteil ergänzt sich daher erst später mit dem Kohlendioxidanteil zu den erwarteten 100 \% (abhängig vom Verweilzeitverhalten des Biogases). Dies ist bei der Auswertung der Messdaten zu berücksichtigen. Aus dem Verlauf der Methankonzentration über der Zeit lassen sich Hinweise über eine günstige Betriebsweise einer kontinuierlich betriebenen Biogasanlage ableiten (maximale Energieausbeute). Aus der jeweiligen Methankonzentration und der Biogasvolumenkurve wird anschließend automatisch das Methan- und damit das Energieertragspotenzial direkt berechnet.

\section{Ausblick}

Das hier vorgestellte Messsystem bieten dem Anwender weitreichende Möglichkeiten zur Untersuchung des Biogas- bzw. Energieertragspotenzials verschiedenster Biomassen. Durch die hohe Messgenauigkeit und gute Reproduzierbarkeut der Ergebnisse werden Optimierungen auf allen Ebenen der Biogaserzeugung (Saatzucht, Verfahrenstechnik, Bioprozesstechnik, Anlagensteuerung und Betreibstechnik usw.) möglich. Die hohe zeitliche Auflösung der Gasentstehung (Methan, Kohlendioxid) ermöglicht erstmals auch Untersuchungen der Kinetik der Biogasentstehung. Beispielsweise konnte bereits im Batch-Versuch der Einfluss der Biomassestabilisierung und Lagerung (Alkoholische Gärung, Milchsäuregärung, Essigsäuregärung etc.) auf die Versäuerungsphase der nachfolgenden Methangärung und die Energie- und Kohlenstoffbilanz des Gesamtprozesses Silierung-Vergärung gezeigt werden. Durch die bisher unerreichte Genauigkeit können nun auch qualitative Hinweise zur Optimierung von kontinuierlichen Vergärungsversuchen gegeben werden z.B. bei der Kalibrierung modellgestützter Prozessführungssysteme zur Effizienzsteigerung von Biogasanlagen. 